\title{
Desenvolvimento de Dashboards para Análise de Características Geográficas e Climáticas do Estado da Paraíba (PB) - Projeto Plataforma Multi-Mapa PB
}

\author{
Miguel Marques Ferreira, Helon David Macêdo Braz, Flavio da Silva Vitorino Gomes, Joseíto de Oliveira Júnior \\ Centro de Energias Alternativas e Renováveis \\ Universidade Federal da Paraíba \\ \{miguel.ferreira; hrlon; flavio; joseito.junior\}@cear.ufpb.br
}

\begin{abstract}
Resumo: O projeto Plataforma Multi-Mapa PB surgiu com o objetivo principal de possibilitar que usuários sem conhecimento profundo em programação, criem dashboards de vários tipos incluindo texto, imagem, listas suspensas, mapas georreferenciados, gráficos e tabelas para visualização de dados. Nesse sentido, um pacote nomeado de json2dash foi desenvolvido e dois dashboards foram elaborados para apresentação e validação da ferramenta utilizando dados obtidos através dos sistemas da National Aeronautics and Space Administration (NASA), da Agência Executiva de Gestão das Águas do Estado da Paraíba (AESA) e da Agência Nacional de Energia Elétrica (ANEEL).
\end{abstract}

Palavras-chave: dashboard; Python; json2dash.

\section{Introdução}

De acordo com dados do IBGE de 2018, o estado da Paraíba tem 1,9\% da população do Brasil, enquanto o seu Produto Interno Bruto (PIB) corresponde a apenas 0,9\% do PIB nacional [1]. Melhorar essa participação não é uma tarefa simples, mas certamente passa pela exploração inteligente de setores em que há um potencial maior inerente ao estado. A geração de energia por meio de fontes renováveis, tais como a solar fotovoltaica e a eólica, são exemplos de áreas com grande potencial de desenvolvimento.

De acordo com a Agência Internacional de Energia Renovável (IRENA), o Brasil é o segundo país no mundo que mais gera emprego no setor de geração de energia elétrica (hidrelétricas, usinas solares e eólicas) [2]. Tais fatores indicam crescimento consistente das fontes renováveis nos próximos anos, encorajando iniciativas no sentido de facilitar o planejamento e operação de novas usinas geradoras.

Em virtude das singularidades de cada região do Brasil, diversos fatores devem ser levados em conta para a exploração de uma fonte de energia. Fatores como a disponibilidade de recursos, interesses comerciais, domínio de tecnologias e a preservação do meio ambiente estão diretamente relacionados com a oferta de energia à população [3].

Dada a importância das características geográficas e climáticas, é imprescindível sua observação, análise e previsão. Nesse contexto, existem órgãos, empresas e agências como a Agência Executiva de Gestão das Águas do Estado da Paraíba (AESA) [4], a Agência Nacional de Energia Elétrica (ANEEL) [5] e a National Aeronautics and Space Administration (NASA) [6] que são responsáveis por mensurar as alterações vigentes e fornecer dados e informações aos mais diversos setores da sociedade.

As formas convencionais de visualização de dados utilizam gráficos, tabelas, diagramas, mapas, infográficos, painéis, etc. Contudo, o uso de dashboards (ou painéis interativos) surge com a proposta de sintetizar, uniformizar e detalhar as informações o máximo possível para evitar quaisquer problemas no entendimento, uma vez que compila os elementos gráficos comuns [7].

A visualização de dados fazendo uso de dashboards analíticos têm como meta a caracterização, predição, ganho de insight e o suporte nos processos de tomadas de decisões [8]. Ainda que existam informações e algumas bases de dados sobre as alterações climáticas, elas não estão disponíveis aos interessados utilizando uma grande variedade de representações gráficas [9].

O projeto Plataforma Multi-Mapa PB surgiu nesse ambiente, tendo como objetivo principal reunir informações relevantes sobre o estado da Paraíba (PB) e disponibilizá-las de forma amigável e simples para a sociedade. Trata-se de uma plataforma web que visa dar aos usuários a possibilidade de desenvolver seus próprios dashboards mesmo sem possuir conhecimento aprofundado de programação. O projeto está na sua primeira etapa, em que o foco é acessar e visualizar dados geográficos e climatológicos.

Em síntese, o presente trabalho expõe o processo de desenvolvimento de dois dashboards. Nessa primeira etapa foram usados dados abertos obtidos por meio dos sistemas da NASA, AESA e ANEEL referentes aos municípios do estado da Paraíba (PB). O trabalho foi organizado em mais quatro seções: Estado da Arte, Metodologia, Desenvolvimento e Resultados, Discussão e Conclusões.

\section{Estado da Arte}

No cenário atual, há vários projetos voltados para a permitir aos usuários a criação de dashboards interativos. Essas implementações se diferenciam pela facilidade de uso, pelo conhecimento de programação requerido e o mais importante, pelo nível de dependência criado entre a ferramenta e o projeto do usuário. Para exemplificar, algumas ferramentas com essa finalidade são Google Data Studio, Grafana, Voila e Panel.

O Google Data Studio é uma ferramenta online que possibilita a criação de dashboards sem a necessidade de utilizar uma linguagem de programação. É totalmente integrada ao ecossistema de aplicações da 
empresa Google, entretanto não é possível implementar um dashboard que execute na máquina local [10].

O Grafana é uma ferramenta online de código aberto que possibilita a elaboração de dashboards sem a necessidade de utilizar uma linguagem de programação. Ao contrário da ferramenta da Google, o Grafana possibilita a integração com APIs Rest, mas também não possibilita implementação na máquina local e não permite a visualização dos dashboards sem um cadastro prévio [11].

Com o Voila é possível transformar de forma rápida e fácil jupyter notebooks em aplicativos web autônomos que podem rodar localmente [12]. O Voila é gratuito de código aberto, mas é necessário o interpretador Python para utilizá-lo.

O Panel é uma biblioteca Python de código aberto que permite criar aplicativos e dashboards, conectando widgets definidos pelo usuário a gráficos, imagens, tabelas ou texto [13]. É possível rodar localmente na máquina do usuário, mas é necessário utilizar o interpretador Python para utilizá-lo.

\section{Metodologia}

O projeto foi implementado a partir do uso da linguagem de programação Python, que é ideal para a concepção de aplicações em muitas áreas graças à sua relativamente fácil curva de aprendizado.

Os valores apresentados nos dashboards de exemplo mostrados neste trabalho foram retirados de uma base de dados local criada pelos integrantes do Projeto MultiMapa PB fazendo uso dos sistemas de dados abertos da AESA, ANEEL e IBGE.

Após a organização dos valores, surgiu a ideia de desenvolver um pacote Python que tivesse a capacidade de auxiliar a visualização web desses dados usando vários componentes. Esse pacote, constituído por um conjunto de módulos, foi nomeado de json2dash. Ele engloba algumas bibliotecas existentes dentro da linguagem Python, visando simplificar processos de programação e, principalmente, remover a necessidade de reescrever comandos comumente utilizados. As principais dependências do pacote json2dash são: Pandas, Folium e Dash.

O pacote Pandas é uma ferramenta desenvolvida para análise prática e manipulação de dados, flexível, de código aberto e baseada em estruturas nomeadas de DataFrames que possibilitam a leitura e escrita de valores tabulados em linhas e colunas, o que a torna bastante rápida e eficiente manipulação de dados [14].

A biblioteca Folium é um invólucro (do inglês, wrapper) de Leaflet.js, uma biblioteca open-source escrita em JavaScript para mapas interativos compatíveis com dispositivos móveis [15]. O Leaflet foi projetado visando a simplicidade, desempenho e usabilidade. Ele funciona com eficiência nas principais plataformas de desktop e móveis, podendo ser estendido com vários plug-ins. Como um wrapper, o Folium herda todas essas características.
O framework Dash, criado com base nas bibliotecas Flask e Plotly, é uma das ferramentas disponíveis para a construção de aplicações analíticas na internet. Renderizado no próprio navegador, sua utilização se torna bastante simples e consiste em uma interface composta por duas partes fundamentais: o layout e as callbacks. Essas partes, compreendem a construção visual com muitos componentes prontos para alocação na página web e funções de retorno para inserção de interatividade aos sites [16].

Dessa forma, a associação das bibliotecas citadas anteriormente originou o json2dash, pacote idealizado com o propósito de eliminar parcialmente a necessidade de utilizar programação para o desenvolvimento de aplicações web, uma vez que o usuário enviará informações por meio de um arquivo de texto no formato JSON (JavaScript Object Notation). As informações fornecidas como entrada são convertidas de forma automática em uma aplicação Dash que pode ser publicada na Internet.

No arquivo enviado pelo utilizador existe a opção de fornecer as chaves principais: callback e layout (Figura 1). Essas informações são suficientes para criação do dashboard.

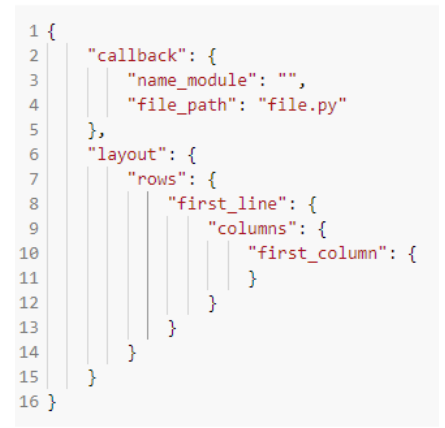

Figura 1. Estrutura de arquivo de texto enviado pelo usuário.

A chave callback é opcional, no entanto ela garante a atualização dinâmica do layout por meio de funcionalidades interativas para os componentes da página web. Quando utilizada, requer o envio do caminho até um arquivo Python com callbacks. A chave layout é obrigatória, sendo utilizada para a construção da parte visual da aplicação. O layout do dashboard é montado utilizando uma grade contendo linhas (rows) e colunas (columns). Além disso, cada coluna pode conter um único componente visual escolhido dentro de uma variedade de elementos, tais como: texto com linguagem de marcação markdown, imagem, lista suspensa de seleção, mapa georreferenciado, gráfico, tabela, etc.

É possível instalar a ferramenta json2dash por meio do instalador de pacotes padrão do Python: o pip. Os membros do projeto direcionam o comando de instalação para o repositório do projeto na nuvem, podendo então desenvolver seus dashboards sem a necessidade de conhecer detalhes de implementação do pacote. 
Para exemplificar as potencialidades do pacote json2dash, dois dashboards exemplo foram elaborados utilizando alguns dos componentes desenvolvidos, com destaque para mapas georreferenciados e gráficos dinâmicos.

\section{Desenvolvimento e Resultados}

Para evidenciar os resultados obtidos até agora, foi desenvolvido um protótipo de dashboard, em sua primeira versão, destinado ao estudo de grandezas climáticas dos municípios do estado da Paraíba utilizando dados de temperatura obtidos por meio do sistema da NASA compreendendo o intervalo de tempo de 1983 até 2018.

Essa aplicação, utilizando sobretudo o componente para geração de gráficos, entrega ao usuário a possibilidade de trabalhar com a seleção de até 13 (treze) tipos básicos de gráfico, incluindo gráficos de pontos, linhas, barras, distribuição (histogramas e boxplots), além de heatmaps (mapas de calor) e dispersão. Além disso, dentre as funcionalidades inseridas na aplicação, é possível manipular as grandezas para os eixos $\mathrm{x}$ e y das figuras, o intervalo de tempo de interesse e até a aparência da figura.

O painel de controle foi dividido em cinco seções: 1) Ranking de cidades por grandeza climática e mesorregião, 2) Gráficos por mesorregião, 3) Gráficos por intervalo de tempo, 4) Gráficos para comparação de grandezas entre cidades e 5) Mapa de calor (HeatMap) para uma cidade exclusiva.

Na Figura 2, é apresentada a segunda seção elaborada com as informações das temperaturas média, mínima e máxima a 2 metros do solo, em ${ }^{\circ} \mathrm{C}$, filtradas a partir da escolha de uma cidade de interesse dentre as mesorregiões da Paraíba: agreste paraibano, Borborema, mata paraibana e sertão paraibano.

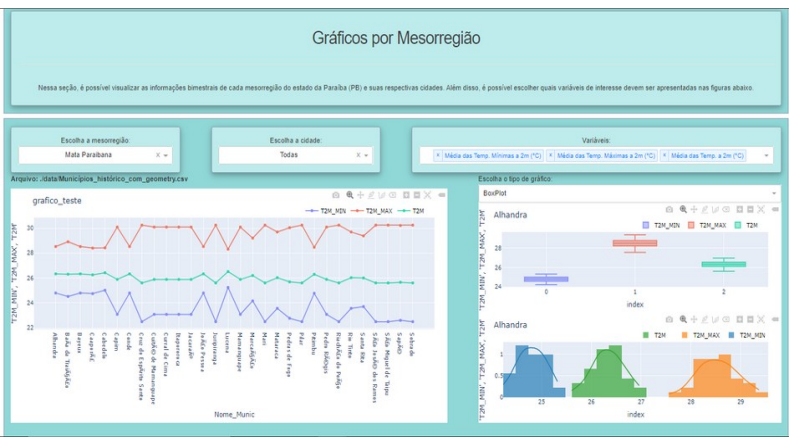

Figura 2. Seção do dashboard: Gráficos por Mesorregião.

Além disso, na maioria das seções, foi fixada a grandeza para o eixo das abscissas (eixo x) com valores relacionados ao intervalo de tempo em que aquela informação foi coletada. E assim, para o eixo das ordenadas (eixo y), múltiplas possibilidades de grandezas estão dispostas para seleção. Por exemplo:
- $\quad$ Precipitações média e acumulada;

- Umidade a 2 metros, específica e relativa;

- Pressão na superfície, em kPa;

- Temperaturas a 2 metros, em ${ }^{\circ} \mathrm{C}$ (mínima, máxima e média);

- Velocidades do Vento a 10 metros e 50 metros, em m/s (mínima, máxima e média).

Outro dashboard com informações georreferenciadas foi criado utilizando dados obtidos da AESA e da ANEEL, os quais dispõem da posição de aerogeradores, geração distribuída e usinas fotovoltaicas além da posição e geométrica de açudes da Paraíba (Figura 3).

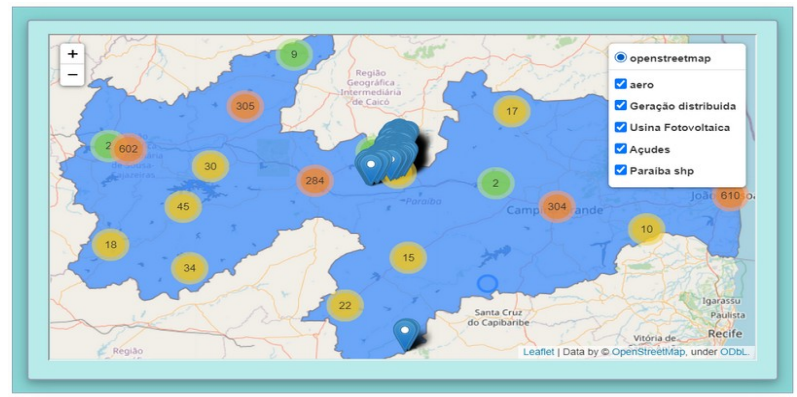

Figura 3. Dashboard com informações georreferenciadas.

\section{Discussão}

Informações a respeito do período de precipitação, incidência solar, regime dos ventos e temperatura são fatores diretamente relacionados com o desempenho de usinas geradoras. Por isso, os dashboards oferecem apoio àqueles que necessitam de informações para fundamentar decisões. O uso de callbacks para a elaboração do painel de controle facilita a realização de diferentes ajustes com complexidade mínima e, assim, a obtenção da visualização conforme desejada.

Na Figura 4, o ranking das 20 cidades da Paraíba com maior média de precipitação desde o ano de 1983 até 2018 é mostrado como um exemplo ilustrativo.

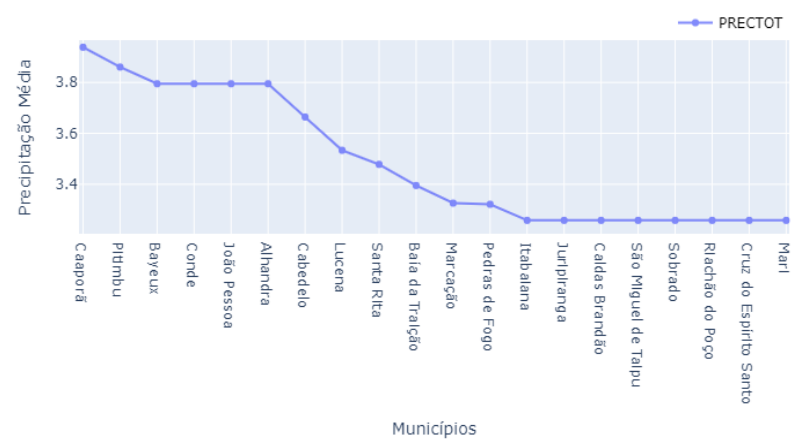

Figura 4. Ranking das cidades da Paraíba pela precipitação média. 
Ainda sobre a Figura 4, é possível perceber que a maioria das cidades que apresentam uma maior precipitação média no intervalo analisado de 35 anos fazem parte da mesorregião da mata paraibana.

Na Figura 5, a temperatura média histórica de cada município, considerando uma distância de 2 metros do solo, é mostrada por meio de um mapa. Nele é possível perceber que a Borborema é a mesorregião em média mais fria, enquanto o sertão paraibano a mais quente.

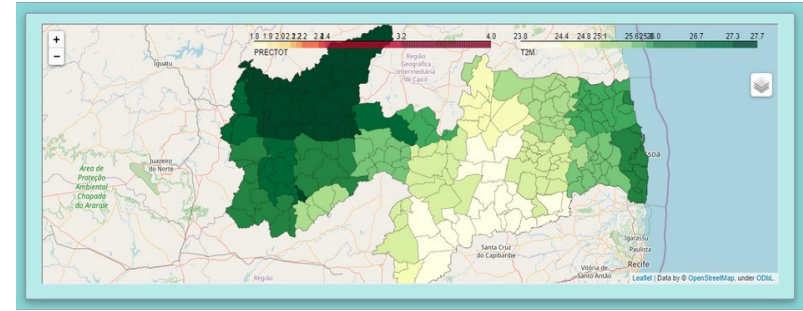

Figura 5. Dashboard com temperatura média a 2 metros do solo.

\section{Conclusões}

O projeto Plataforma Multi-Mapa PB foi apresentado do ponto de vista de sua motivação e objetivos gerais, tendo sido detalhada apenas sua primeira etapa, que consistiu no desenvolvimento de um pacote Python nomeado de json2dash e de dois dashboards de validação, voltadas para grandezas de interesse geográficas e climáticas do estado da Paraíba.

Foram utilizados dados abertos de diferentes fontes e apenas alguns dos componentes de visualização de informações disponíveis no pacote desenvolvido, mas considerando que esses resultados iniciais foram obtidos com um mínimo de programação por parte dos usuários, ficou demonstrado o potencial do projeto em termos de democratização do acesso a dados de forma genérica e também do ponto de vista das oportunidades saindo do contexto de visualização de dados e partindo para análises e previsões.

\section{Bibliografia}

[1] Coordenação de Contas Nacionais. (2020) Sistema de contas regionais: Brasil: 2018/IBGE, Coordenação de Contas Nacionais, 2018. IBGE.

[2] IRENA. International Renewable Energy Agency. ( 2020) Renewable Energy and Jobs - Annual Review, 2020. [s.n.]. Disponível em: <https://www.irena.org/ publications/2020/Sep/Renewable-Energy-and-JobsAnnual-Review-2020>. Acesso em 27/03/2021.

[3] Farias, M.; Sellitto, A. (2011) Uso da energia ao longo da história: evolução e perspectivas futuras. Revista Liberato 12(17): 7-16. DOI: 10.31514/rliberato.2011v12n17.p07

[4] AESA (2021) Informações Básicas - AESA. Disponível em: <http://www.aesa.pb.gov.br/>. Acesso em 27/03/2021.

[5] ANEEL (2021). Conheça a ANEEL. Disponível em: $<$ https://www.aneel.gov.br/a-aneel>. Acesso em 27/03/21.

[6] NASA (2021) POWER Project. Disponível em: $<$ https://power.larc.nasa.gov/>. Acesso em 27/03/21.

[7] Rolim, D. (2020) Dashboards para desenvolvimento de aplicações e visualização de dados para plataformas de cidades inteligentes. Dissertação de Mestrado. Universidade Federal do Rio Grande do Norte.

[8] Okoh, C.; Roy, R.; Mehnen, J. (2017) Maintenance informatics dashboard design for through-life engineering services. Procedia CIRP 59: 166-171.

[9] Sousa, M. (2019)Visualização de dados climáticos na Web. Tese de Doutorado. Universidade de Coimbra.

[10] Google (2021) Google Analytics Solutions. Disponível em: <https://datastudio.withgoogle.com/>. Acesso em 22/06/2021.

[11] Grafana (2021) Grafana: The open observability plataform. Projeto online. Disponível em: https://grafana.com/. Acesso em: 25/06/21.

[12] Voilá (2021) voila-dasboards/voila. Projeto online. Disponível em: <https://github.com/ voila-dashboards/voila> . Acesso em 25/06/21.

[13] Panel (2021) holoviz/panel. Projeto online. Disponível em: <https://github.com/holoviz/panel>. Acesso em: 25/06/21.

[14] Pandas.org. (2021) About pandas. Disponível em: $<$ https://pandas.pydata.org/about/>. Acesso em 27 março 2021.

[15] Leafletjs.org. (2021) Leaflet: An open-source JavaScript library for mobile-friendly interactive maps. Disponível em: <https://leafletjs.com/>. Acesso em 27/03/2021.

[16] Dash Enterprise (org.) (2021) Introduction to Dash. Disponível em: <https://dash.plotly.com/ introduction>. Acesso em 27/03/21. 